\title{
Island Shape-Induced Transition from 2D to 3D Growth for Pt/Pt(111)
}

\author{
Jacobsen, Joachim; Jacobsen, Karsten Wedel; Stoltze, Per; Nørskov, Jens Kehlet
}

Published in:

Physical Review Letters

Link to article, DOI:

10.1103/PhysRevLett.74.2295

Publication date:

1995

Document Version

Publisher's PDF, also known as Version of record

Link back to DTU Orbit

Citation (APA):

Jacobsen, J., Jacobsen, K. W., Stoltze, P., \& Nørskov, J. K. (1995). Island Shape-Induced Transition from 2D to 3D Growth for Pt/Pt(111). Physical Review Letters, 74(12), 2295-2298.

https://doi.org/10.1103/PhysRevLett.74.2295

\section{General rights}

Copyright and moral rights for the publications made accessible in the public portal are retained by the authors and/or other copyright owners and it is a condition of accessing publications that users recognise and abide by the legal requirements associated with these rights.

- Users may download and print one copy of any publication from the public portal for the purpose of private study or research.

- You may not further distribute the material or use it for any profit-making activity or commercial gain

- You may freely distribute the URL identifying the publication in the public portal

If you believe that this document breaches copyright please contact us providing details, and we will remove access to the work immediately and investigate your claim. 


\title{
Island Shape-Induced Transition from 2D to 3D Growth for Pt/Pt(111)
}

\author{
Joachim Jacobsen, Karsten W. Jacobsen, Per Stoltze, and Jens K. Nørskov \\ Center for Atomic-scale Materials Physics and Physics Department, Technical University of Denmark, DK-2800 Lyngby, Denmark
}

(Received 1 August 1994; revised manuscript received 25 January 1995)

\begin{abstract}
We present a kinetic Monte Carlo simulation of the growth of $\mathrm{Pt}$ on $\mathrm{Pt}(111)$ capable of describing the experimentally observed temperature dependence of the island shapes and the growth mode. We show that the transition from a $2 \mathrm{D}$ growth mode at low temperatures to a $3 \mathrm{D}$ mode at higher temperatures is closely related to the disappearance of kink sites and the appearance of the triangular islands observed in the 3D growth regime.
\end{abstract}

PACS numbers: $68.55 . \mathrm{Jk}, 68.35 . \mathrm{Bs}$

The nucleation and growth of metal overlayers on metal surfaces provide a significant challenge to our understanding of surfaces. It is the combination of many different surface processes that determine the island shape, the density of islands, and the growth mode, that is, whether the growth proceeds in a layer-by-layer (2D) mode or a rough three-dimensional (3D) overlayer is formed. If eventually we become able to control the shapes of the islands on the surface and the growth mode, we will be in a completely new position to "design" surfaces with particular mechanical, electrical, magnetic, or chemical properties.

Even the simplest homoepitaxial systems are still not understood. For Pt deposited on $\mathrm{Pt}(111)$, for instance, it is observed that as the temperature is lowered, the growth mode changes first from $2 \mathrm{D}$ to $3 \mathrm{D}$, but then back to $2 \mathrm{D}$ at even lower temperatures [1-3]. The first transition is expected. It is well established that the diffusion barrier down from an island is larger than the barrier for diffusion on the flat surface [4-7]. Below some temperature this process freezes out. The atoms landing on top of an already existing island cannot diffuse down and attach at the edge, but instead nucleate new islands on top of the old one. The reentrant $2 \mathrm{D}$ growth at even lower temperatures is much harder to understand. What is it that makes the diffusion down from islands possible again at temperatures as low as $100-200 \mathrm{~K}$ ?

There has been a large number of suggestions for mechanisms of the reentrant growth in the literature. It has been suggested that since the islands are smaller and more irregular at low temperatures, either the size [1] or the availability of kink sites [1,3] affect the barrier for descent. Support for the size dependence has been provided by $\mathrm{Li}$ and DePristo [5] based on approximate total energy calculations within the corrected effective medium theory, while calculations of Villarba and Jonsson [8] using the embedded atom method have pointed out that very special exchange processes next to kinks can have a much lower barrier than any other down-diffusion processes. Other ideas in the field include the concept of "transient mobility" [9] and "downward funneling" [10]. While some of these ideas may be important, it remains to directly evaluate their importance for the growth process. A very elegant analysis by
Tersoff, van der Gon, and Tromp have shown how the concept of a critical island size may be useful in a discussion of 2D vs 3D growth [11]. In a full kinetic Monte Carlo simulation, Smilauer, Wilby, and Vvedensky [12] are able to obtain the reentrant growth transition; however, the barriers used are not relevant for $\mathrm{Pt} / \mathrm{Pt}(111)$.

In the present Letter we suggest that the low temperature transition between 2D and 3D growth is driven by the change in both size and shape of the islands with temperature. We base our conclusions on a set of kinetic Monte Carlo simulations of the growth of $\mathrm{Pt}$ on $\operatorname{Pt}(111)$ as a function of temperature. We show that with a realistic set of parameters consistent with a number of independent experimental observations and with detailed calculations of diffusion processes using the effective medium theory [13], we can reproduce the observed reentrant behavior. We show that while the low temperature 2D growth is related to the low barrier diffusion processes at kinks which are abundant on the rough islands at this temperature, at higher temperatures the 3D behavior is closely connected to the occurrence of triangular islands. Some kinks are still present at the edges of these islands, but the fast diffusion process down from islands is solely found at kinks on the step orientations that disappear when the triangular islands form.

The kinetic Monte Carlo model we use is similar to the one proposed by Voter [14]. The simulations are done on an fcc (111) lattice [15]. In agreement with molecular dynamics simulations [5,6], atoms arriving from the gas phase stick when they are supported by three atoms in the lower layer (i.e., we neglect overhangs, and funneling is to some extent included).

The input into the simulations is a set of activation barriers and prefactors for the many possible diffusion processes. In principle the number of different barriers is very large, and their exact values are unknown. Only approximate total energy methods can at present give results for all the processes of interest here. We have used the effective medium theory (EMT) to give us an idea about the important processes in analogy with the analysis of Liu et al. [16]. This way we can limit the number of distinct processes in the model by making a classification depending on the local environment. The EMT barriers 
are not expected to be sufficiently accurate to describe the growth process. However, if we assume the relative ordering of the barriers to be reasonably correct, we can construct a growth model by scaling the EMT barriers to get a good description of the experiment.

Figure 1 summarizes the results for a number of different diffusion processes over an island, down from an island, along the two different $A$ and $B$ steps, and away from an island. A large number of the features seen in Fig. 1 are in qualitative agreement with experimental observations, in particular from field ion microscopy (FIM) $[4,17]$ and with the detailed local density functional calculations for $\mathrm{Al} / \mathrm{Al}(111)$ [7].

From calculations for a large number of different diffusion processes, we have found that we can systematize our calculated barriers for in-layer processes by noting that the barrier depends on the number of in-layer neighbors in the initial state, and on whether or not the process is a dissociation away from an island.

It has been shown by Liu et al. [18] that to explain the island shapes at higher temperatures it is important to distinguish between the $A$ and $B$ steps. In the temperature range of interest here, where triangular islands having $A$ steps are observed, it is sufficient to include the asymmetry at corners of $A$ and $B$ steps (position $B$ in Fig. 1).

Villarba and Jonsson [8] have pointed out the importance of kink sites for diffusion down from islands. As illustrated in Fig. 2, we also find that sites next to kinks have the lowest barrier for down diffusion through an exchange process. But we find that a low barrier process only exists on the $B$ steps. On the $A$ steps the atom that moves out from the step does not have an easy path between two atoms underneath, but has to move over an
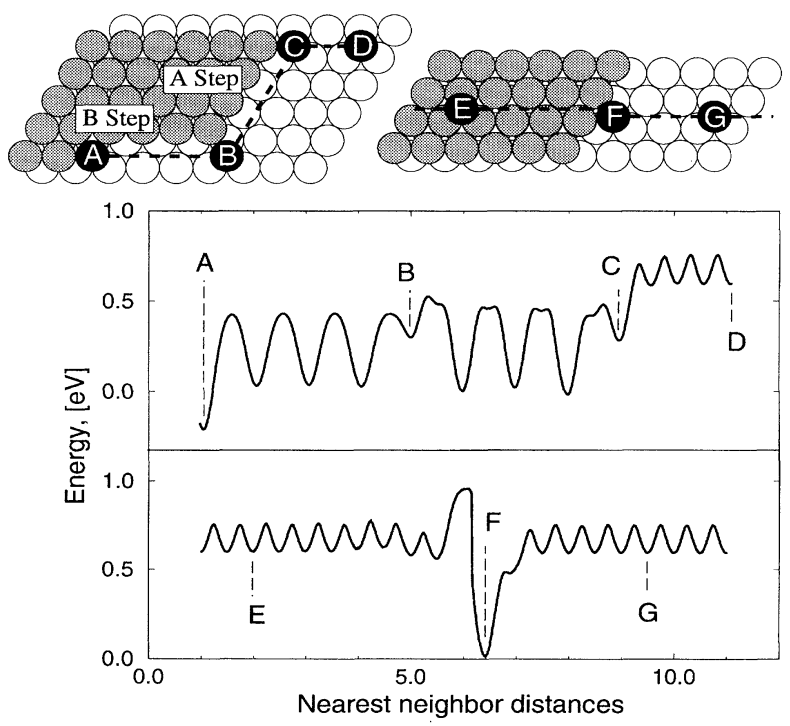

FIG. 1. Examples of effective medium calculations. An atom is dragged along the indicated path, and the energy is minimized with respect to all other degrees of freedom. Only part of the unit cell used in the calculations is shown.

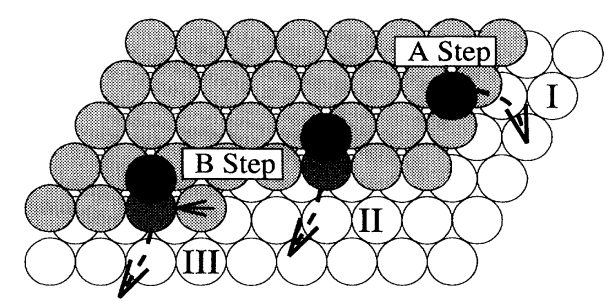

FIG. 2. The three interlayer processes included in Table I. In the transition state of process III, the kink atom is shifted to the left, lowering the energy barrier [8].

on-top site (cf. Fig. 2). The calculated barriers for the intralayer and interlayer diffusion processes are summarized in Table $\mathrm{I}$.

As expected, if we use the EMT barriers directly in a kinetic Monte Carlo simulation of the initial stages of growth, we get a poor agreement with the experiment. In such a simulation too few islands are nucleated. Also these islands take a much too regular shape. However, choosing a prefactor of $10^{11} \mathrm{~s}^{-1}$, and scaling all barriers for the intralayer processes up by a factor of 1.6 [19], we obtain a growth model qualitatively and quantitatively in agreement with the experimentally observed island densities and shapes [2,3]. Also, the resulting model value for the terrace diffusion $(0.26 \mathrm{eV})$ agrees very well with a direct measurement of the diffusion rate at $100 \mathrm{~K}$ by Kellogg and Feibelman using the FIM [20]. They obtain $0.25 \pm 0.02 \mathrm{eV}$ assuming a prefactor of $10^{12} \mathrm{~s}^{-1}$. The fact that the EMT barriers appear to be approximately $30 \%$ too low is a very reasonable accuracy for the approximate total energy method. It should be compared to the fact that a full local density functional calculation of the barrier for the normal diffusion process gave a barrier of $0.38 \mathrm{eV}$ [20].

When continuing the growth simulation with the deposition of several monolayers, we also find that the EMT barriers for the interlayer processes are too low to obtain $3 \mathrm{D}$ behavior at any temperature above $250 \mathrm{~K}$ whether or not the kink process is included. In the model presented in Table I, exhibiting 3D growth above $350 \mathrm{~K}$ and reentrant 2D at lower temperatures, we have increased the barrier for down diffusion at straight steps by a factor of 1.6 as for the intralayer processes and the barrier for the kink process by a factor 1.5 , and chosen the same prefactor for all processes.

It should be stressed that we have tried to choose a common scaling for the barriers for reasons of simplicity, and not because we believe in the existence of an exact scaling between the EMT and the true barriers. Nor is our result critically dependent on the exact value of the barriers in the model. The important point is to obtain reasonable island densities and shapes. Only one barrier is crucial, and this is the barrier for down diffusion at kink sites. The rate of this particular process has a very strong influence on the growth mode around the transition temperature, and we had to choose a slightly different scaling factor for this barrier. 
TABLE I. EMT energy barriers in $\mathrm{eV}$ and the adjusted values used in the model simulation. $N_{i}\left(N_{f}\right)$ is the number of in-layer nearest neighbors in the initial (final) state. For given $N_{i}$ and $N_{f}$ the EMT calculations are performed for a variety of local environments. The corresponding energy interval of the barriers justifies the classification. The interlayer processes are illustrated in Fig. 2.

\begin{tabular}{|c|c|c|c|c|}
\hline \multicolumn{5}{|c|}{ In-layer processes } \\
\hline Process type & $N_{i}$ & $N_{f}$ & EMT & Model \\
\hline Diffusion on terrace & 0 & 0 & 0.16 & \\
\hline Diffusion to step & 0 & $\geq 1$ & $0.12-0.15^{\}}$ & 0.26 \\
\hline Diffusion from top of edge toward island & 0 & 0 & 0.20 & 0.32 \\
\hline Diffusion along $A$ step & 1 & $\geq 1$ & $0.23-0.25$ & 0.37 \\
\hline Diffusion along $B$ step & 1 & $\geq 1$ & $0.18-0.20$ & 0.29 \\
\hline Diffusion along step & 2 & $\geq 1$ & $0.4-0.45$ & 0.64 \\
\hline Diffusion along step & 3 & $\geq 1$ & $0.63-0.65$ & 1.01 \\
\hline Diffusion along step & $\geq 4$ & $\geq 1$ & $\geq 0.83$ & $\infty$ \\
\hline Dissociation from step & 1 & 0 & $0.50-0.60$ & 0.80 \\
\hline Dissociation from step & 2 & 0 & $0.71-0.74$ & $\infty$ \\
\hline Dissociation from step & $\geq 3$ & 0 & $\geq 0.96$ & $\infty$ \\
\hline \multicolumn{5}{|c|}{ Interlayer processes } \\
\hline Overedge at $A$ step, I & & & 0.41 & 0.59 \\
\hline Exchange at $B$ step, II & & & 0.37 & \\
\hline Exchange next to kink at $B$ step, III & & & 0.26 & 0.39 \\
\hline
\end{tabular}

We then turn to the simulation of the growth process. In the upper part of Fig. 3 we show the calculated island structure after deposition of 0.3 monolayer of $\mathrm{Pt}$ on $\mathrm{Pt}(111)$ at two different temperatures. Both the density and the shapes are very close to the scanning tunneling microscopy observations of Bott, Michely, and Comsa [2], confirming the validity of the model. To characterize the nature of the growth process we monitor the density of steps as a function of the growth time. For a 2D growth pattern we expect the step density to oscillate, while it should increase monotonically for 3D growth. The step density is closely related to the He scattering intensity which is used experimentally to characterize the growth mode [1]. Figure 4 shows the step density at 255,270 , and $370 \mathrm{~K}$. At $370 \mathrm{~K}$ the growth mode is clearly $3 \mathrm{D}$, while it is much more $2 \mathrm{D}$-like at the lower temperatures. In agreement with experimental findings the 2D character increases with temperature up to the transition temperature. In Fig. 3 we also include a snapshot of the surface after 4 monolayers have been deposited. At $370 \mathrm{~K}$ six layers are exposed, and the 3D nature of the growth is evident. At $255 \mathrm{~K}$, the growth is much more layer by layer like, but it is not a perfect $2 \mathrm{D}$ growth. Three layers are open at a time. The nonperfect nature of the low temperature 2D growth is also reflected in the fact that the oscillations in the step density are seen to decay.

The simulation gives us some insight into the important factors determining whether $2 \mathrm{D}$ or $3 \mathrm{D}$ growth is observed. In our model the low temperature $2 \mathrm{D}$ growth is a direct result of the very low barrier for descent at kinks. If this process is not included, we have found no set of barriers and prefactors consistent with the other experimental observations that are able to give the reentrant $2 \mathrm{D}$ growth mode.
As the temperature is increased the Pt islands become larger, and the step density is decreased. The diffusion along the steps speeds up and decreases the kink density. In our simulations another thing happens, too, as the temperature is increased. The asymmetry of the diffusion around corners of islands and the predominant interlayer diffusion at the $B$ steps give rise to a change of the island shape. The islands do not become (quasi)hexagonal but rather triangular, because more atoms go to the $B$ step than to the $A$ step (cf. Fig. 1). The $B$ steps essentially disappear, and since it is only the $B$ steps that have the low barrier

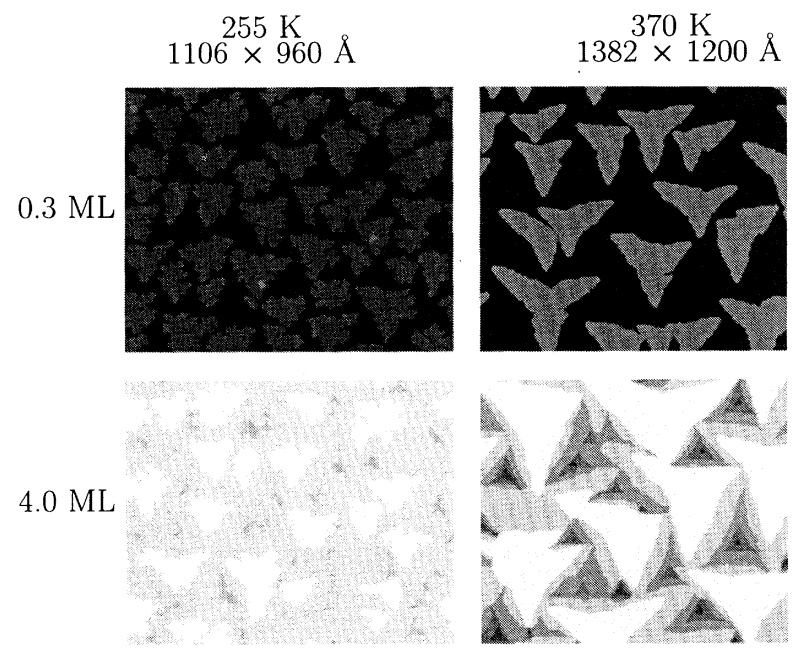

FIG. 3. Top view of the obtained surfaces after the deposition of 0.3 and $4.0 \mathrm{ML}$ at a rate of $0.0033 \mathrm{ML} / \mathrm{s}$, and at a temperature below and above the low temperature growth mode transition. The grey scale indicates the height above the surface. 


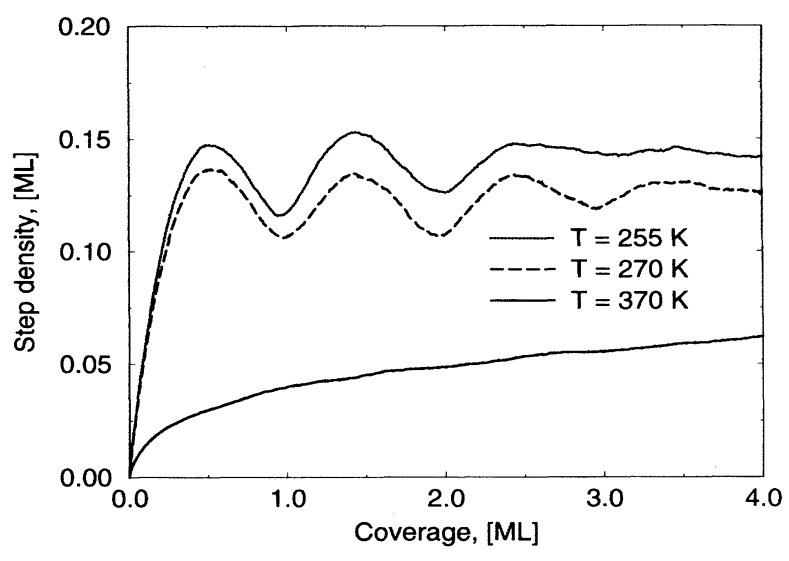

FIG. 4. The step density as a function of coverage at three temperatures. The deposition rate is $0.0033 \mathrm{ML} / \mathrm{s}$.

kink sites, the diffusion down from the islands is reduced further.

If we do not include any asymmetry between the $A$ and $B$ steps, the islands grow quasihexagonal, and even the relatively few available kinks on these compact islands make the 3D growth hard to obtain. We can only get a low temperature $2 \mathrm{D}$ and high temperature $3 \mathrm{D}$ growth if we reduce the calculated barrier at the kink site to $0.24 \mathrm{eV}$ and choose an extremely low prefactor for this process of $10^{8} \mathrm{~s}^{-1}$. Given that the EMT consistently underestimates diffusion barriers, this seems very unlikely. When we include the asymmetry we can choose the more reasonable parameters, but the most direct justification of the included difference between kinks at $A$ and $B$ steps comes from observing the influence of this on the islands' shape at 0.3 monolayer (ML). All the interlayer transport occurs at kinks at $B$ steps, since the other available processes are frozen out. In the $3 \mathrm{D}$ regime it means that it occurs at the corners of the triangular islands. The effect at $0.3 \mathrm{ML}$ is that the corners grow faster than the edges, yielding concave curved triangles and the "fjords" in the triangles that are very consistent with the island shapes observed with scanning tunneling microscopy. Also note the experimental observation that the temperature range of the intermediate $3 \mathrm{D}$ growth coincides exactly with the temperature range where the islands are compact and triangular having $A$ steps.

In conclusion, we have constructed a model including the important diffusion processes for $\mathrm{Pt}$ on clean and island covered Pt(111) surfaces. Using the kinetic Monte Carlo method the model can describe in great detail the observed island shapes in the temperature range $200-370 \mathrm{~K}$ as well as the transition between $2 \mathrm{D}$ and $3 \mathrm{D}$ growth around $340 \mathrm{~K}$. The simulations point unambiguously to the importance of kink sites for the diffusion of adatoms down from an island as proposed previously [3]. As a new effect we show that the transition in the growth mode is related to a transition in shape of the islands from irregular at low temperatures to triangular at the higher temperatures.
The present work underlines the fact that even a qualitative understanding of the growth modes requires a detailed understanding of the underlying diffusion processes. The strong dependence of the barriers for diffusion on the local geometry gives rise to strong asymmetries in the diffusion rates which ultimately determine the growth mode.

We thank G. Comsa for many stimulating discussions. The Center for Atomic-scale Materials Physics is sponsored by The Danish National Research Foundation.

[1] R. Kunkel, B. Poelsema, L. K. Verheij, and G. Comsa, Phys. Rev. Lett. 65, 733 (1990).

[2] M. Bott, T. Michely, and G. Comsa, Surf. Sci. 272, 161 (1992).

[3] M. Henzler, T. Schmidt, and E.Z. Luo, The Structure of Surfaces IV (World Scientific, Singapore, 1994).

[4] For a recent review of the field ion microscopy studies of single atom motion on metal surfaces, see, e.g., G. Erlich, Surf. Sci. 299/300, 628 (1994), and references therein.

[5] Y. Li and A. E. DePristo (to be published).

[6] P. Stoltze and J.K. Nørskov, Phys. Rev. B 48, 5607 (1993).

[7] R. Stumpf and M. Scheffler, Surf. Sci. 306-309, 501 (1994).

[8] M. Villarba and H. Jonsson, Phys. Rev. B 49, 2208 (1994); Surf. Sci. 317, 15 (1994).

[9] W.F. Egelhoff and I. Jacob, Phys. Rev. Lett. 62, 921 (1989).

[10] J. W. Evans, D. E. Sanders, P. A. Thiel, and A. E. DePristo, Phys. Rev. B 41, 5410 (1990).

[11] J. Tersoff, A. W. Dernier van der Gon, and R. M. Tromp, Phys. Rev. Lett. 72, 266 (1994).

[12] P. Smilauer, M. R. Wilby, and D. Vvdensky, Phys. Rev. B 47, 4119 (1993).

[13] K. W. Jacobsen, J. K. Nørskov, and M. J. Puska, Phys. Rev. B 35, 7423 (1987). The electron density used was taken from a self-consistent solution for $\mathrm{Pt}$ in jellium, while the remaining three parameters were determined from the measured cohesive energy, bulk modulus, and shear modulus. The parameters for $\mathrm{Pt}$ and the other fcc metals and test calculations can be found in P. Stoltze, J. Phys. Condens. Matter 6, 9495 (1994).

[14] A. F. Voter, Phys. Rev. B 34, 6819 (1986).

[15] The fact that the diffusion takes place via the hcp sites is included in the prefactor of the rate by multiplying it by $1 / 3$.

[16] S. Liu, Z. Zhang, J. Nørskov, and H. Metiu, Surf. Sci. 321, 161 (1994).

[17] S.C. Wang and G. Erlich, Phys. Rev. Lett. 67, 2509 (1991).

[18] S. Liu, Z. Zhang, G. Comsa, and H. Metiu, Phys. Rev. Lett. 71, 2967 (1993).

[19] Since the model only has a limited number of distinct processes, to each of these there will be a range of EMT barriers. The scaling is then from the lowest EMT barrier for a process of the given type.

[20] P. J. Feibelman, J.S. Nelson, and G. L. Kellogg, Phys. Rev. B 49, 10548 (1994). 
$255 \mathrm{~K}$ $1106 \times 960 \AA$
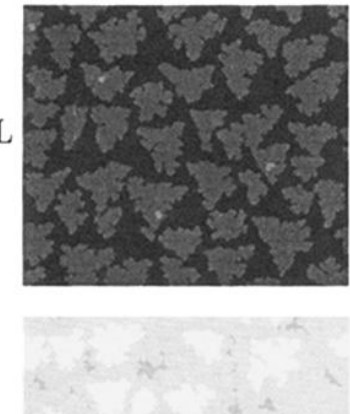

4.0 ML

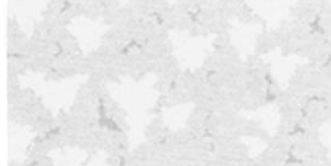

$370 \mathrm{~K}$ $1382 \times 1200 \AA$
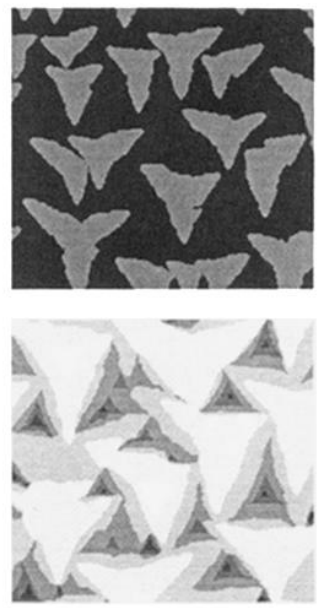

FIG. 3. Top view of the obtained surfaces after the deposition of 0.3 and $4.0 \mathrm{ML}$ at a rate of $0.0033 \mathrm{ML} / \mathrm{s}$, and at a temperature below and above the low temperature growth mode transition. The grey scale indicates the height above the surface. 